\title{
Gewalt in der Intensivpflege
}

— Warum wird jemand, der einen Beruf ergreift, um anderen zu helfen, plötzlich gewalttätig gegenüber seinen Patienten? Alexandra Mende, Fachkrankenpflegerin in der Medizinischen Universitätsklinik Heidel- berg, hat dem Tabuthema "Gewalt in der Intensivpflege" die Abschlussarbeit ihrer Fachweiterbildung Anästhesie und Intensivpflege gewidmet. Für die ausführliche Auseinandersetzung mit Ursachen und

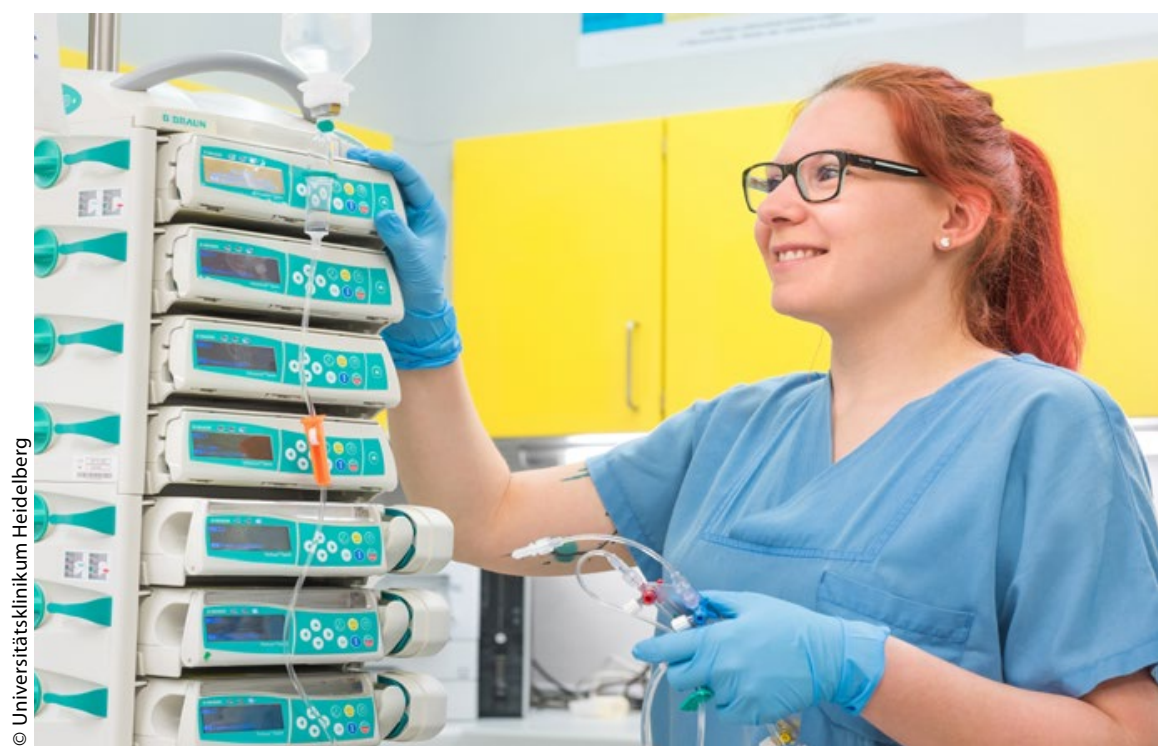

Alexandra Mende, Fachkrankenpflegerin an der Medizinischen Uniklinik Heidelberg
Prävention wurde sie jetzt auf dem 27. Symposium für Intensivpflege und Intensivmedizin mit dem Hanse-Pflegepreis ausgezeichnet. „Wir haben eine große Verantwortung für unsere intensivpflichtigen Patienten. Umso wichtiger ist es, auch vor unangenehmen Themen wie Gewalt nicht die Augen zu verschließen", erklärt Edgar Reisch, Pflegedirektor am Universitätsklinikum Heidelberg.

Frustration und Stress hat Alexandra Mende als wichtige Einflussfaktoren für die Entstehung von Gewalt identifiziert: So können negative Entwicklungen im Team, Personalmangel und Stress Aggression hervorrufen. Hinzu kommt ein Machtgefälle zwischen Pflegekraft und Patient. „Ein komatöser Patient in intensivmedizinischer Behandlung befindet sich unweigerlich in einer hilflosen Situation", sagt Mende. "Solche Situationen erleichtern es, aktiv oder passiv Gewalt auszuüben." Ob Menschen unter solchen Umständen aggressiv werden, hängt auch von persönlichen Faktoren ab. Mende spricht sich dafür aus, Pflegende zu sensibilisieren und das Thema bereits in die Ausbildungsmodule zu integrieren, wie es an der Akademie für Gesundheitsberufe Heidelberg bereits geschieht.

\section{Wissenschaftliche Umfrage \\ Teilnehmer gesucht}

— Um die Anerkennung und Darstellung einer Berufsgruppe, die aktuell im Fokus der Medien steht, geht es in der Dissertation von Gabriele Tauber, Physiotherapeutin und Doktorandin an der Universität Paderborn im Bereich Arbeits- und Organisationspsychologie (Prof. Dr. Niclas Schaper). Um neue Möglichkeiten für den Berufstand der Pflegekräfte zu erforschen, untersucht sie im Rahmen einer wissenschaftlichen Befragung, welche Arbeitsanforderungen und unterstützenden Bedingungen Einfluss auf die Motivation einer Pflegekraft haben. Machen Sie mit! Der Fragebogen kann online oder in Papierform ausgefüllt werden.

www.gabitauber.de
Info- und Kontaktstudientag

\section{Vorzüge der Pflegestudiengänge kennenlernen}

— Am 21.04.2017 lädt die Pflegewissenschaftliche Fakultät der Philosophisch-Theologischen Hochschule Vallendar (PTHV) wieder zum Informations- und Kontaktstudientag unter dem Motto "Sie + Wir = Zukunft!". An dem „Tag der offenen Tür“" werden von 10 bis 15 Uhr profunde Einblicke in die bewährten Studienprogramme des Masterstudiums und des Promotionsprogramms Pflegewissenschaft gegeben. Zudem wird der Lehramtsstudiengang "Pflege an Berufsbildenden Schulen", den die PTHV gemeinsam mit der Universität Koblenz-Landau anbietet, sowie der Bachelorstudiengang ,Pflegeexpertise' vorgestellt, der sich an Pflegekräfte in der Praxis richtet.

Lehrende, Mitarbeiter, Studierende und Referendare der PTHV geben Einblicke in Studienziele, Lehrinhalte, Betreuungsangebote und das studentische Leben in Vallendar und Koblenz. In zahlreichen Kurzvorträgen werden Inhalte und Ziele von Hauptmodulen und Lehrveranstaltungen vorgestellt. Zudem wird es ausreichend Zeit für Gespräche und persönliche Begegnungen mit Dozenten und Studierenden der Pflegewissenschaftlichen Fakultät sowie individuelle Beratungsgespräche geben. Bewerbungen sind für alle Studiengänge ab sofort möglich, außer für den Lehramtsstudiengang (ab 01.06.2017). 